\title{
The Importance of Remaining Empathic
}

\author{
Constantin Filip*
}

The patient-physician relationship has many dimensions - technical, psychological, moral and legal. At its essence, however, it is a professional relationship. As in all such relationships, there exists a set of boundaries between the parties that allow each to act according to his particular role and responsibilities, in a safe environment. The existence of such boundaries implies that the notion of "professional distance" is, in fact, a necessary one in clinical practice.

However, "distance" can be mistakenly equated with "detachment" - or the removal of the affective component from the patient-physician relationship. "Detachment" and "engagement" are two discrete approaches to dealing with patients. The latter, in the author's view, is the only approach which truly allows for empathy. This essay will attempt to characterize empathic engagement in the physician-patient relationship, and will aim to reveal the advantages of this approach over detachment.

In a famous address given to his students, Osler proclaims that a "judicious measure of obtuseness" - or detachment - should be developed upon entering the field of medicine (1). This so-called "art of detachment" represented for him the way physicians could meet the exigencies of the profession with "firmness and courage" (1). Although Osler is also well known for his superior ability to engage with patients, his teachings on the subject of detachment are not adopted by today's medical schools. According to Carr, "one problem with detachment [...] is that its essential starting point is separation. Rather than communicating care and acceptance to the patient, the physician who begins with detachment communicates impatience, nonchalance, or perhaps even [...] contempt." (2) Other authors consider that detachment is a flawed model for conducting patient care. Halpern (3) reviews at least two major

\footnotetext{
*To whom correspondence should be addressed: Constantin Filip constantin.filip@mail.mcgill.ca
}

reasons why doctors themselves might seek detachment from, rather than engage emotionally with, their patients: first, the belief that emotions are inherently subjective and interfere with objectivity; and second, an alleged protection from burnout. Halpern refutes the first point by showing how the arrival of new technologies over the last century have contributed to undermine "humanistic" practice in favour of strictly "objective" measures of care. She then shows that the skill of clinical empathy is in fact "emotional reasoning". In her view, the clinician uses subjective, experiential input for specific, cognitive aims to enhance medical judgement (3). Secondly, she argues that, despite popular belief, detachment does not protect doctors from burnout (3); rather, she asserts that burnout can be linked to time pressures and other organisational issues that interfere with doctor-patient relationships. Communication with the patient on an emotional level, on the contrary, seems to play a beneficial role in physician satisfaction.

However, one must not confuse clinical empathy with the psychological defence mechanism of projection. The common definition of empathy, "projecting one's personality into (and so fully comprehending) the object of contemplation", may not be advisable clinically. As some critics have pointed out, empathy risks blurring the line between self and other (4). Recognizing the impact of over-involvement in medicine, Figley outlined the concept of "compassion fatigue" - an entity considered separate from burnout (5). It is believed that its cause may be an over-intensive identification with the survival strategies adopted by patients, and inappropriate or lacking doctor survival strategies (5).

Thus, this essay refers to the term "empathy", not to describe imagining oneself in the patient's position, but to mean engaging with the patients' emotional experience in order to better comprehend specific aspects of their worldview. Halpern argues that such empathy can help doctors better focus their attention on what is humanly significant as it facilitates trust and disclosure from patients (6). 
As a first-year medical student at McGill, I am reminded that there are important concerns within society about today's doctors; namely, even though they may be excellent technicians, doctors fail to meet patients' expectations with regard to interpersonal communication. For example, during a class discussion in our Physicianship course, many students narrated with frustration their worst experiences at the doctor's office. Among the stories were several accounts of encounters with doctors who did not establish any connection whatsoever; while barely making any eye contact, they appeared "too cold," "too busy," or certainly too detached for their patients' comfort.

To be empathic, physicians must be ready to be moved by their patients' recounting of their problems and concerns. Although their work may at times feel overwhelming and some patients may not be fully pleased with them, doctors must recognize that each patient is entitled to some of their attention, which includes an emotional presence. Of course, it would be completely unreasonable for physicians to allow their emotions to permeate their sessions with patients. Nonetheless, when a physician adopts a strictly detached stance toward his patient, the patient is less likely to receive the appropriate nonverbal feedback from the interaction. One can argue that such feedback may yet be given by an emotionally detached physician, who may have insight into the emotional state of the patient. However, as is the case in day-to-day conversations, patients can be very good at noticing when conversation feedback is not emotionally genuine-because many verbal or nonverbal cues may be out of sync-and this "acting" is likely to engender frustrations, leading to a poor patient-physician relationship.

As a general rule, it is not adequate that doctors train themselves to provide "generic" feedback that merely mimics concern. Rather, it is important to individualize one's therapeutic style to suit patients' needs. As Halpern illustrates (3), some people respond best to reassurance, others to acknowledging the legitimacy of their fears, and others to a more confident, authoritative style. To achieve this finer understanding and knowledge of the appropriateness of each situation, the patient-physician relationship needs to have a genuine emotional component, which rests upon physician empathy.

Finally, one of the most compelling reasons to emphasize empathy in clinical medicine comes from its relationship to healing. Although there are few studies that look at this association directly, there is consistent evidence that physicians who display a warm, friendly, and reassuring manner with their patients are more effective healers (7). For instance, a randomized trial involving 133 homeless adults found that such "compassionate care" increased patient satisfaction and reduced the frequency of re-admissions to the emergency department (8). In another study which involved 230 consecutive hospital consultations, patients' perception of the doctor's empathy was found to be strongly correlated with their ability to cope with, and understand, their illness (9). Hence, there is a healing power to emotional communication, as the literature suggests. Also, it seems that the actual nature of the illness itself is not a limiting factor, as the variables examined in these studies vary substantially from childhood asthma symptoms, to HIV progression markers, to heart failure occurence (3). This appears to support the notion that physicians in all fields of medicine can enhance their healing by adopting a more empathic, supportive approach to their patients. ${ }^{1}$

In conclusion, while "professional distance" remains a necessary factor in the patient-physician relationship, detachment appears to be a less desirable approach to patient contact than empathic engagement. Detachment seems to be wrongly advocated as a tool for maintaining objectivity and preventing burnout. Furthermore, a "generic" type of empathy appears to engender more frustration than it actually serves patients. Most significantly, there is empirical evidence that associates an empathic approach to positive healing outcomes. Therefore, to suit patients' needs, physicians must individualize their styles and remain attuned to emotional communication.

The ability to empathically attune one's attention to a variety of patients is a demanding task, but a necessary one. Maintaining an open mind instead of developing obtuseness is perhaps a better way for medical students to develop empathy.

\section{REFERENCES}

1. Osler W. Aequanimitas, with other addresses to medical students, nurses and practitioners of medicine [Internet]. Baltimore: The Johns Hopkins Health System; 1999 [updated 1999; cited Feb 7, 2009]; Available from: http://www.medicalarchives.jhmi.edu/osler/aequessay.htm.

2. Carr MF. Who Cares About Empathy? Second Opinion. 2001 July; 7 .

3. Halpern J. From detached concern to empathy : humanizing medical practice. Oxford: Oxford University Press; 2001.

4. Edwards KA. Critiquing empathy : insights for professional education. Second Opinion. 2001 Feb;4.

5. Figley CR. Compassion fatigue : coping with secondary traumatic stress disorder in those who treat the traumatized [e-

\footnotetext{
${ }^{1}$ One possibility to further understanding this matter would be to conduct a study that contrasted patient outcomes between an empathic group of treating physicians and a detached one. Such studies may possibly provide further evidence for the usefulness of empathy in medicine.
} 
book]. New York: Brunner/Mazel; 1995. Chapter 1, An overview; p. 1-20. [cited 2009 Feb 21]. Available from: Psychology Press.

6. Halpern J. What is clinical empathy? J Gen Int Med. [Perspectives]. 2003 Aug; 18:670-4. 165p.

7. Di Blasi Z, Harkness E, Ernst E, Georgiou A, Kleijnen J. Influence of context effects on health outcomes: A systematic review. Lancet. 2001 March 10;357:757-62.
8. Redelmeier DA, Molin JP, Tibshirani RJ. A randomised trial of compassionate care for the homeless in an emergency department. Lancet. 1995 May 6;345:1131-4.

9. Mercer SW, Watt GCM, Reilly D. Empathy is important for enablement. BMJ. 2001 April 7;322:865.

Constantin Filip (M.D.C.M. 2012) is currently a medical student at McGill University. 